\title{
Multivalley transport and the integer quantum Hall effect in a PbTe quantum well
}

\author{
V. A. Chitta, ${ }^{1}$ W. Desrat, ${ }^{2}$ D. K. Maude, ${ }^{2}$ B. A. Piot,${ }^{2}$ N. F. Oliveira, Jr., ${ }^{1}$ P. H. O. Rappl, ${ }^{3}$ A. Y. Ueta, ${ }^{3}$ and E. Abramof ${ }^{3}$ \\ ${ }^{1}$ Instituto de Fisica, Universidade de São Paulo, CP 66318, 05315-970 São Paulo, SP, Brazil \\ ${ }^{2}$ Grenoble High Magnetic Field Laboratory, CNRS, Boîte Postale 166, 38042 Grenoble Cedex 9, France \\ ${ }^{3}$ Laboratório Associado de Sensores e Materiais, Instituto Nacional de Pesquisas Espaciais, CP 515, \\ 12201-970 São José dos Campos, SP, Brazil
}

(Received 23 June 2005; revised manuscript received 9 September 2005; published 17 November 2005)

\begin{abstract}
We report magnetotransport measurements performed on a $10 \mathrm{~nm} n$-type $\mathrm{PbTe} / \mathrm{Pb}_{0.9} \mathrm{Eu}_{0.1} \mathrm{Te}$ quantum well grown on a $\mathrm{BaF}_{2}$ substrate. In contrast to previous work, both the Hall and longitudinal resistances show a clearly defined signature of the integer quantum Hall effect with no sign of parallel conduction. Due to the large carrier density, the occupation of two longitudinal subbands and one oblique valley subband leads to an unusual sequence of filling factors.
\end{abstract}

DOI: 10.1103/PhysRevB.72.195326

\section{INTRODUCTION}

Narrow gap IV-VI semiconductors are widely used to fabricate infrared lasers and detectors. Among them, lead telluride $(\mathrm{PbTe})$ possesses a complex band structure characterized by nonparabolic and strongly anisotropic multiple valleys. Early investigations on this compound have been devoted to magneto-optical studies performed on several-micrometerthick films. ${ }^{1-5}$ Surprisingly, very few transport measurements have been performed on this system at reduced dimensionality. ${ }^{6,7}$ Continuing progress in growing IV-VI heterostructures by molecular-beam epitaxy (MBE) now gives access to high-quality quantum wells, making them alternative candidates for the fabrication of two-dimensional electron gases (2DEGs). Mobilities as large as $2 \times 10^{6} \mathrm{~cm}^{2} / \mathrm{V} \mathrm{s}$ have been reported in PbTe films. ${ }^{8}$

Lead telluride is a narrow gap semiconductor $\left(E_{g}\right.$ $=0.19 \mathrm{eV})$ whose Bravais lattice is face-centered cubic. ${ }^{9}$ The Fermi surface consists of four equivalent strongly anisotropic ellipsoids of revolution $\left(K=m_{\|} / m_{\perp} \sim 10\right)$ with their main axis along the $\langle 111\rangle$ directions. For a heterostructure grown along the [111] crystallographic direction, however, this fourfold degeneracy is generally lifted due to strain and/or confinement. Therefore, the large anisotropy and multivalley nature are expected to give rise to a complex density of states, leading to a nontrivial behavior of the electronic transport as a function of the magnetic field.

$\mathrm{PbTe}$ has a high-frequency dielectric constant $\varepsilon_{\infty} \approx 36$ at $T=77 \mathrm{~K},{ }^{10}$ approximately three times larger than that of GaAs. PbTe is therefore a potential model system for studying the quantum Hall effect with a greatly reduced influence of electron-electron interactions. Due to the proximity of a ferroelectric phase transition at low temperature, $\mathrm{PbTe}$ has a large static dielectric constant $\varepsilon_{0} \approx 1400$ at $T=4.2 \mathrm{~K} .{ }^{10}$ The resulting effective screening of charged impurities, defects, and dislocations is directly responsible for the high electron mobilities reported in PbTe. The large dielectric constant has also been shown to modify significantly the electron localization and phase breaking mechanisms, ${ }^{11}$ while the possible observation of fractional quantum Hall states in this system remains an open question. Indeed, measurements in $\mathrm{PbTe}$ quantum point contacts suggest that electron-electron inter-
PACS number(s): 73.43.Qt, 73.21.Fg, 71.20.Nr, 71.70.Di

actions may still play a role despite the large dielectric constant. ${ }^{12}$

In this paper, we investigate the quantum Hall effect in a narrow $\mathrm{PbTe} / \mathrm{Pb}_{0.9} \mathrm{Eu}_{0.1} \mathrm{Te}$ quantum well at low temperatures. Both the longitudinal and Hall resistances show clear signatures of the integer quantum Hall effect with, in contrast to previous studies, ${ }^{13,14}$ a complete absence of parallel conduction. From the temperature dependence of the magnetoresistance minima, the size of the energy gaps is obtained. Using the Mitchell and Wallis model, the density of states of the 2DEG, including the effect of nonparabolicity, is used to calculate the transport properties. In this way, the magneticfield dependence of the longitudinal and Hall resistances is reproduced with a good qualitative agreement. Due to the large carrier density, there are three subbands occupied (two longitudinal and one oblique valley), which naturally explains the unconventional sequence of filling factors observed experimentally. Finally, magnetoresistance data in tilted field are presented and discussed.

\section{EXPERIMENT}

The sample investigated in this work is a 10 -nm-wide $\mathrm{PbTe}$ quantum well grown by molecular-beam epitaxy on a [111]-oriented barium fluoride $\left(\mathrm{BaF}_{2}\right)$ substrate. Before growing the quantum-well structure, a 1.5 - $\mu \mathrm{m}$-thick buffer layer of $\mathrm{Pb}_{1-x} \mathrm{Eu}_{x} \mathrm{Te}$ with $x=0.1$ is grown to accommodate the $4.8 \%$ lattice misfit and reduce the undesirable effects of the dislocations which appear at the interface during the initial stage of growth. Subsequently, the quantum-well structure consisting of a $10 \mathrm{~nm}$ PbTe layer embedded between two $25 \mathrm{~nm} \mathrm{~Pb}{ }_{0.9} \mathrm{Eu}_{0.1}$ Te barriers is grown. The Eu content of the buffer layer, which is assumed to be the same as for the barriers, was determined from the transmission spectrum measured at room temperature in a Fourier transform spectrometer.

High-resolution x-ray-diffraction analysis was used to determine the well and barrier widths and revealed that the quantum-well stack has the same in-plane lattice constant as the buffer layer (coherent growth). Therefore, the PbTe well is completely strained with a tensile in-plane biaxial strain. The large europium content $(x=10 \%)$ of the barriers ensures 


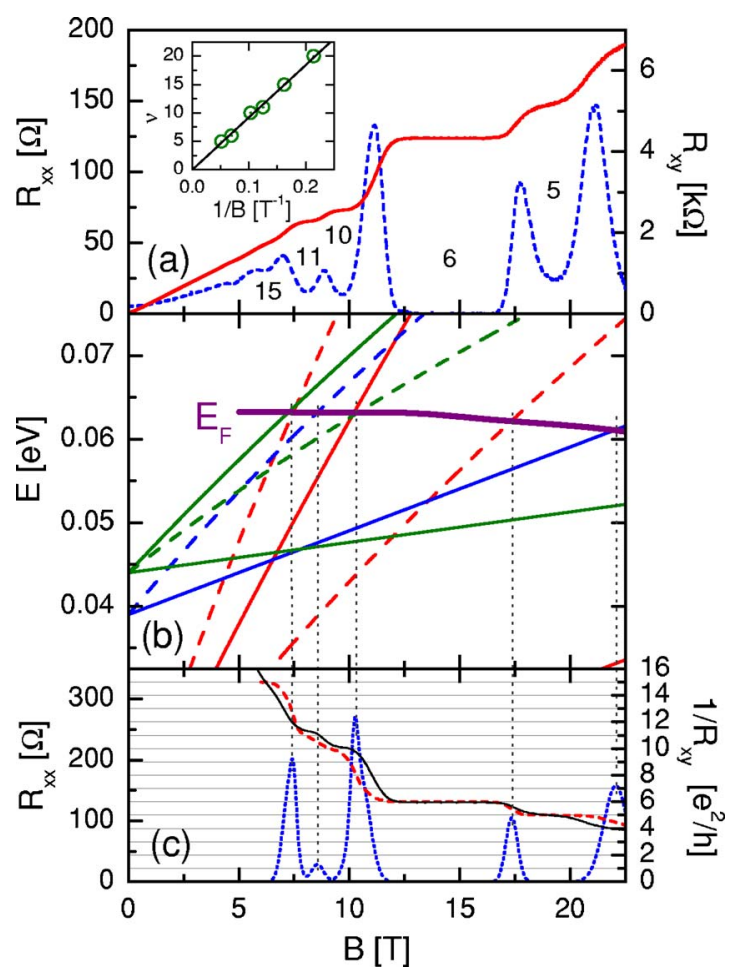

FIG. 1. (Color online) (a) Magnetoresistance $\left(R_{x x}\right.$, dashed line) and Hall resistance $\left(R_{x y}\right.$, solid line) as a function of the perpendicular magnetic field. The inset shows the filling factor vs the inverse magnetic field position of the minima in $R_{x x}$. (b) Fan chart of the low-energy spin-split Landau levels vs $B$. The first longitudinal, second longitudinal, and first oblique subbands are represented with the solid/dashed lines associated with the down/up spins. The thick solid line is the calculated position of the Fermi energy. (c) Computed magnetoresistance (dotted curve) and inverse Hall resistance (dashed curve) as a function of the magnetic field. The thin $1 / R_{x y}$ solid curve is the experimental data.

a good confinement of the electrons in the well and reduces considerably the risk of parallel conduction since the carrier density and mobility of PbEuTe decrease drastically with increasing Eu concentration. ${ }^{13}$ The barrier on the substrate side and the quantum well are both doped with bismuth from a $\mathrm{Bi}_{2} \mathrm{Te}_{3}$ source. The $\mathrm{Bi}$ donor level has been shown to be resonant with the conduction band. ${ }^{15}$ For electrical measurements, thin gold wires are soldered with indium in order to form Ohmic contacts to the two-dimensional electron gas in a Van der Pauw geometry.

The transport measurements have been carried out either in a dilution fridge, where the sample is placed directly in the mixing chamber $(T=30 \mathrm{mK})$, or in a variable temperature insert (VTI) for the higher-temperature measurements. Resistances are measured in a four-wire configuration using a low quasi-dc current $(I=0.1-2 \mu \mathrm{A}$ and $f=10.66 \mathrm{~Hz})$ and standard phase-sensitive detection.

\section{MAGNETOTRANSPORT}

Figure 1(a) shows the longitudinal resistance $\left(R_{x x}\right)$ and Hall resistance $\left(R_{x y}\right)$ as a function of the perpendicular magnetic field at $T=80 \mathrm{mK}$. The oscillating behavior of $R_{x x}$ ver- sus $B$ as well as the presence of plateaus in $R_{x y}$ indicate the well developed quantization of the density of states, which demonstrates the 2D nature of the electron gas. It confirms the efficient confinement by the $\mathrm{Pb}_{0.9} \mathrm{Eu}_{0.1}$ Te barriers. More specifically, around $B=15 \mathrm{~T}$ a clear signature of dissipationless conduction is observed. $R_{x x}$ presents a wide zero resistance region and $R_{x y}$ a corresponding flat plateau, which is exactly quantized, within experimental error, to $h / 6 e^{2}$ [see Fig. 1(c)]. To our knowledge, this is the first time that the integer quantum Hall effect (QHE) has been observed in a $\mathrm{PbTe}$ quantum well with no sign of parallel conduction. Therefore, unlike previous samples, the buffer layer and barriers of our heterostructure are fully depleted and at low temperature the electronic transport takes place exclusively in the PbTe quantum well. ${ }^{13}$

In order to assign filling factors to the observed plateaus, we plot the inverse Hall resistance in units of $e^{2} / h$ as shown in Fig. 1(c). From this graph, the filling factors can be read directly from the right axis. They obey the following decreasing sequence: $\nu=15,11,10,6,5,4$. By plotting these filling factors as a function of the inverse magnetic fields at which the corresponding $R_{x x}$ minima occur [open circles in the inset of Fig. 1(a)], one finds the expected linear dependence. From the slope of the least-squares fit, we obtain a carrier density of $n_{s}=(2.23 \pm 0.03) \times 10^{12} \mathrm{~cm}^{-2}$, which is very close to the value estimated from the slope of the low-field Hall resistance equal to $(2.28 \pm 0.02) \times 10^{12} \mathrm{~cm}^{-2}$. The very good agreement between carrier densities obtained by both methods further confirms that the conduction takes place only in the PbTe quantum well, with no other parallel conduction channel. Finally, from the zero-field resistivity, we estimate the electron mobility to be $2.7 \times 10^{5} \mathrm{~cm}^{2} / \mathrm{V} \mathrm{s}$.

Figure 2(c) represents the magnetoresistance for different temperatures from 4 to $76 \mathrm{~K}$. As expected, the $R_{x x}$ minima are less developed at higher temperatures. The Arrhenius plot in Fig. 2(a) shows the resistance minima normalized to the zero-field resistance $\left(R_{0}\right)$ plotted on a log scale as a function of the inverse temperature for the three integer filling factors $\nu=10,6$, and 5 , appearing at the respective magnetic fields $B=10.2,15.7$, and $20.4 \mathrm{~T}$. The slope of the least-squares fits provides an estimate of the mobility gaps assuming a thermally activated transport regime, $R_{x x} \propto \exp \left(-E_{a} / 2 k_{B} T\right)$. We obtain $E_{a}=1.55 \pm 0.12,5.31 \pm 0.09$, and $1.43 \pm 0.03 \mathrm{meV}$ for $\nu=10,6$, and 5 , respectively. For the two even filling factors 10 and 6, the Lifshitz-Kosevich (LK) formula, i.e., $\Delta R_{x x} / R_{\max } \sim X / \sinh (X)$ with $X=2 \pi^{2} k T / \Delta$, can also be satisfactorily applied as shown in Fig. 2(b). It gives energy gaps $\Delta=8.11 \pm 0.77$ and $18.3 \pm 1.8 \mathrm{meV}$, respectively. The activation energy corresponds to the mobility gap, while the LK gap corresponds to the energetic separation between the Landau-level centers. The measured LK gaps are much less than the cyclotron energy reflecting the large electronic $g$ factor and the anisotropic mass of the different occupied valleys in our PbTe quantum well.

From the temperature dependence of the zero-field resistance, we can plot the evolution of the Drude mobility, inversely proportional to $R_{0}$, as a function of temperature. This is represented on a log-log graph in the inset of Fig. 2(c). The mobility increases as the temperature is reduced and satu- 

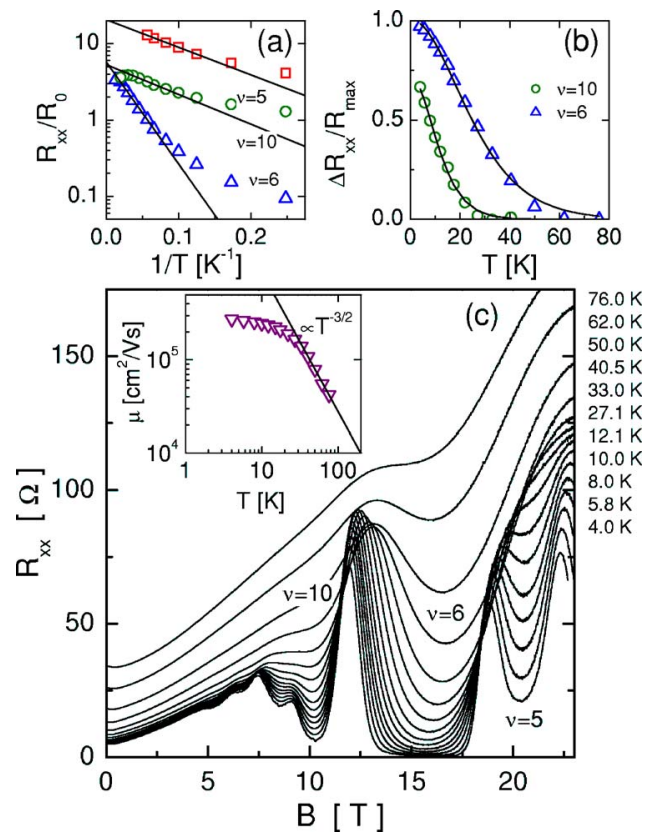

FIG. 2. (Color online) (a) Arrhenius plot for filling factors $\nu$ $=10,6$, and 5 occurring at $B=10.2,15.7$, and $20.4 \mathrm{~T}$. The solid lines are least-squares fits. (b) The same data plotted as $\Delta R_{x x} / R_{\max }$ vs $T$ for $\nu=10$ and 6 . The solid lines are least-squares fits to the LifshitzKosevich formula as described in the text. (c) Magnetic-field dependence of the longitudinal resistance $R_{x x}$ for increasing temperatures from 4 to $76 \mathrm{~K}$. The inset shows the mobility as a function of the temperature (note the log scales for both axes). The solid line shows a $T^{-3 / 2}$ dependence.

rates at low temperature around $270000 \mathrm{~cm}^{2} / \mathrm{V} \mathrm{s}$. For $T$ $>30 \mathrm{~K}$, the mobility scales as $T^{-3 / 2}$ due to phonon scattering.

\section{THEORETICAL MODEL}

We now propose a simple density of states model in order to reproduce the magnetotransport data, in particular to verify if the unexpected sequence of filling factors can be reproduced by taking into account both the spin and valley degeneracies of the system. We underline that Springholz et al. ${ }^{14}$ adopted a similar approach to describe their QHE data. However, the lower carrier density in their system simplified the analysis considerably, since only one valley subband was occupied.

Before presenting the model, we recall that the direct gap between the valence and conduction bands in PbTe occurs at the intersection of the [111] direction with the Brillouin zone face-the $L$ point. For a heterostructure grown along the [111] crystallographic direction, this fourfold degeneracy is lifted at low temperatures due to strain arising from the lat- tice mismatch between the well and barrier materials and the different thermal-expansion coefficients between the IV-VI semiconductor structure and the $\mathrm{BaF}_{2}$ substrate. This leads to a typical splitting of a few meV (Refs. 17 and 18) between the singly degenerate longitudinal valley, whose main ellipsoid axis lies along the [111] direction, and the three equivalent oblique valleys tilted away from the [111] direction by an angle $\phi=70.53^{\circ}$. In narrow quantum wells, however, this valley splitting is dominated by the large difference in the confinement energies between the longitudinal and threefolddegenerate oblique valleys arising from the strong anisotropy $\left(m_{\|} / m_{\perp} \sim 10\right)$.

In order to calculate these confinement energies, the confinement masses of the longitudinal and oblique valleys have to be computed first. Theoretical models for the $L$ conduction-band electronic effective-mass ellipsoids are already available in the literature. Here, we use the $\mathbf{k} \cdot \mathbf{p}$ theory developed by Mitchell and Wallis in which the interaction between the lowest conduction band and highest valence band is treated exactly, while the four more distant bands are considered using a $k^{2}$ approximation. The conduction bandedge values of the effective masses are given by ${ }^{3,19}$

$$
\frac{m_{e}}{m_{\|, \perp}}=\left(\frac{2 P_{\|, \perp}^{2}}{m_{e}}\right) \frac{1}{E_{g}}+\frac{m_{e}}{m_{l, t}^{-}},
$$

where the parameters are given in Table $\mathrm{I}$ and $m_{e}$ is the free-electron mass. The calculated values for valley masses are given in Table II.

From geometrical arguments, the confinement masses along the $z$ axis $(z \|[111])$ are $m_{\ell}^{\text {conf }}=m_{\|}$and $m_{o}^{\text {conf }}$ $=9 m_{\|} m_{\perp} /\left(8 m_{\|}+m_{\perp}\right){ }^{20}$ The $\ell$ and $o$ subscripts stand for the longitudinal and oblique valleys, respectively. The cyclotron mass given by the projection of the mass ellipsoid on the $x-y$ plane is given for each valley by $m_{\ell}^{\text {cyc }}=m_{\perp}$ and $m_{o}^{\text {cyc }}$ $=\frac{1}{3} m_{\perp}\left(1+8 m_{\|} / m_{\perp}\right)^{1 / 2}$. The calculated effective masses for the well and barrier are summarized in Table II.

We are now able to calculate the confinement energies of the longitudinal and oblique valleys in our $10 \mathrm{~nm}$ PbTe quantum well. We refer to the work of Andrada e Silva ${ }^{21}$ and Abramof et al. ${ }^{17}$ where the confinement energy levels are solutions of the following equation:

$$
\frac{\chi}{m_{b}}=\frac{q}{m_{w}} \tan \left(\frac{q L}{2}\right)
$$

for the even states and where the tangent function is replaced by - cot for the odd solutions. $L$ is the well width, and $q$ $=\sqrt{\left(2 m_{w} / \hbar^{2}\right) E}$ and $\chi=\sqrt{\left(2 m_{b} / \hbar^{2}\right)\left(\Delta E_{c}-E\right)}$ are the wave vectors in the well and the barriers, respectively. $\Delta E_{c}$ is the barrier height, i.e., the conduction-band offset.

TABLE I. Band parameters of PbTe in Mitchell and Wallis notation. From Bauer et al. ${ }^{16}$ Masses are expressed in units of $m_{e}$.

\begin{tabular}{cccccccc}
\hline \hline$E_{g}$ & $2 P_{\|}^{2} / m_{e}$ & $P_{\perp} / P_{\|}$ & $m_{l}^{-}$ & $m_{t}^{-}$ & $m_{t}^{+}$ & $g_{l}^{-}$ & $g_{l}^{+}$ \\
\hline $0.19 \mathrm{eV}$ & $6.02 \mathrm{eV}$ & 3.42 & 0.505 & 0.060 & -0.102 & 1.72 & -2.61 \\
\hline \hline
\end{tabular}


TABLE II. Summary of the band parameters for the well and barrier materials. The band parameters for $\mathrm{Pb}_{0.9} \mathrm{Eu}_{0.1} \mathrm{Te}$ are from Prinz et al. ${ }^{11}$ The projected confinement and cyclotron masses (in units of $m_{e}$ ) for longitudinal and oblique valleys for the investigated [111] quantum well are shown.

\begin{tabular}{cccccccc}
\hline \hline & $E_{g}(\mathrm{eV})$ & $m_{\perp}$ & $m_{\|}$ & $m_{\ell}^{\text {conf }}$ & $m_{\ell}^{\text {cyc }}$ & $m_{o}^{\text {conf }}$ & $m_{o}^{\text {cyc }}$ \\
\hline $\mathrm{PbTe}$ & 0.190 & 0.021 & 0.213 & 0.231 & 0.021 & 0.023 & 0.063 \\
$\mathrm{~Pb}_{0.9} \mathrm{Eu}_{0.1} \mathrm{Te}$ & 0.638 & 0.066 & 0.536 & $0.536^{\mathrm{a}}$ & & $0.073^{\mathrm{a}}$ & \\
\hline
\end{tabular}

${ }^{a}$ Mass used to calculate the evanescent wave function in the barrier.

Using the band parameters and the projected confinement masses given in Table II and assuming a 55:45 conductionto valence-band offset ${ }^{22}$ so that $\Delta E_{c}=0.246 \mathrm{eV}$, we find that the subband energies, with respect to the conduction-band edge, are $11,39,79,125,173$, and $221 \mathrm{meV}$ for the longitudinal valley, and 44 and $154 \mathrm{meV}$ for the oblique valleys, which have a lighter confinement mass.

Integrating over the two-dimensional density of states for all possible subbands to obtain the measured carrier density, we find a Fermi energy of $63 \mathrm{meV}$. For this calculation, we consider that each subband is $\alpha_{\ell, o} m_{\ell, o}^{\mathrm{cyc}} / \pi \hbar^{2}$ degenerate, where $\alpha_{\ell, o}$ and $m_{\ell, o}^{\mathrm{cyc}}$ are the subband degeneracy (1 and 3) and electronic cyclotron mass of the longitudinal and oblique valleys given in Table II. Thus, three subbands are occupied: the first longitudinal subband $(E=11 \mathrm{meV})$, the second longitudinal subband $(E=39 \mathrm{meV})$, and the first oblique subband $(E=44 \mathrm{meV})$. Note that in the following, we neglect the valley splitting due to strain, as a reasonable estimate for this splitting is $\delta E_{g}^{\ell}-\delta E_{g}^{o} \approx 1.5 \mathrm{meV}$, which is significantly smaller than the confinement energy scale and is therefore unable to change the order of subbands in the quantum well. ${ }^{18}$

In the presence of a magnetic field, each of these subbands splits into spin-resolved Landau levels, whose following expressions are derived from the exact solutions of the 3 D case when $\vec{B} \|[111]:{ }^{1}$

$$
\begin{aligned}
E_{i, n, \uparrow}= & E_{i}+\frac{1}{2}\left(a_{n}+b_{n}\right) \\
& +\frac{1}{2}\left[\left(a_{n}-b_{n}\right)^{2}+4 \hbar \frac{e B}{m_{e}}\left(\frac{2 P_{\perp}^{2}}{m_{e}}\right)(n+1)\right]^{1 / 2}, \\
E_{i, n, \downarrow}= & E_{i}+\frac{1}{2}\left(c_{n-1}+d_{n-1}\right) \\
& +\frac{1}{2}\left[\left(c_{n-1}-d_{n-1}\right)^{2}+4 \hbar \frac{e B}{m_{e}}\left(\frac{2 P_{\perp}^{2}}{m_{e}}\right) n\right]^{1 / 2},
\end{aligned}
$$

where $E_{i}$ are the confinement energies already computed, $n$ $=0,1,2, \ldots$ is the orbital quantum number, $\uparrow / \downarrow$ stand for the $\sigma= \pm \frac{1}{2}$ spin states, and

$$
\begin{aligned}
& a_{n}=\frac{1}{2} E_{g}+\hbar \omega_{t}^{-}\left(n+\frac{1}{2}\right)+\frac{1}{2} g_{l}^{-} \mu_{B} B, \\
& b_{n}=-\frac{1}{2} E_{g}+\hbar \omega_{t}^{+}\left(n+\frac{3}{2}\right)-\frac{1}{2} g_{l}^{+} \mu_{B} B,
\end{aligned}
$$

$$
\begin{aligned}
& c_{n}=\frac{1}{2} E_{g}+\hbar \omega_{t}^{-}\left(n+\frac{3}{2}\right)-\frac{1}{2} g_{l}^{-} \mu_{B} B, \\
& d_{n}=-\frac{1}{2} E_{g}+\hbar \omega_{t}^{+}\left(n+\frac{1}{2}\right)+\frac{1}{2} g_{l}^{+} \mu_{B} B
\end{aligned}
$$

with $\omega_{t}^{ \pm}=e B / m_{t}^{ \pm}$. In this Mitchell and Wallis approach, the energy of the Landau levels depends on the far-band contributions to the transverse masses $m_{t}^{ \pm}$and longitudinal $g$ factors $g_{l}^{ \pm}$in the valence $(+)$and conduction (-) bands. The $2 P_{\perp}^{2} / m_{e}$ term arises from the direct interaction between the valence and conduction bands.

Note that Eqs. (3) and (4) include the nonparabolicity effect which is relevant at high fields for narrow-gap semiconductors. This leads to sublinear magnetic-field-dependent Landau levels. In their initial form, Eqs. (3) and (4) are exact solutions of the $3 \mathrm{D}$ case with the magnetic field applied along the [111] direction. ${ }^{1,3,6}$ In our 2D system, we use the same expressions for the longitudinal and oblique valleys for the sake of simplicity.

Figure 1(b) shows the resulting Landau-level fan chart, where the lowest first longitudinal, second longitudinal, and first oblique subbands are represented. In this picture, the theoretical zero-field values are maintained unchanged (i.e., 11,39 , and $44 \mathrm{meV}$, respectively) as well as three of the well established values of the far-band contributions $m_{t}^{+}, g_{l}^{-}$, and $g_{l}^{+}$given in Table I, kept fixed for all three subbands. Conversely, the far-band contribution of the electronic transverse effective mass $m_{t}^{-}$and the conduction-band momentum matrix element $2 P_{\perp}^{2} / m_{e}$ are treated as fitting parameters in order to reproduce the experimental data as accurately as possible. For the self-consistent computation of the magnetic-field dependence of the Fermi energy, Gaussian broadened Landau levels are considered,

$$
D(E)=\alpha \frac{e B}{h} \frac{1}{\Gamma \sqrt{2 \pi}} \exp \left[\frac{-\left(E-E_{i, n, \uparrow / \downarrow}\right)^{2}}{2 \Gamma^{2}}\right],
$$

where an empirical broadening parameter $\Gamma=10 \mathrm{meV}$ independent of the valley is used. The degeneracy $\alpha$ is equal to 1 and 3 for the longitudinal and oblique valleys, respectively. By taking the integral over the density of states to be equal to the carrier density, one obtains the Fermi energy, which is plotted in Fig. 1(b). The calculated Fermi energy is quasiconstant as a result of the large broadening of the Gaussian Landau levels. Since the longitudinal resistance is proportional to the density of states at the Fermi energy, a maximum in $R_{x x}(B)$ occurs each time $E_{F}$ coincides with the center of a Landau level, as represented by the thin vertical dotted 
lines. In order to obtain the theoretical density of states and the calculated $R_{x x}$ trace, the following values of $m_{t}^{-}$and $2 P_{\perp}^{2} / m_{e}$ have been used in the simulation: $m_{t}^{-}=0.055,0.055$, and $0.14 m_{e}$ and $2 P_{\perp}^{2} / m_{e}=4.3,3.4$, and $2.7 \mathrm{eV}$ for the first, second, and third subbands, respectively. We underline that these values are not reliable but remain acceptable with respect to the theoretical parameters summarized in Table I. However, they provide a realistic order of magnitude for the nonparabolicity and the resulting nonlinearity of the Landau levels versus $B$. It is important to note that in this simple approach, strain effects are neglected.

Despite its limitations, our simple model provides valuable insight and allows us to explain the unusual sequence of filling factors deduced from the quantized plateaus in $R_{x y}$. Referring to Fig. 1(b), we can easily understand the transfer of electrons between the longitudinal and oblique valleys. For instance at $\nu=5$, three levels are occupied: two singly degenerate longitudinal levels and one threefold degenerate oblique level. The total filling factor is equal to the sum over the filling factors of the longitudinal and oblique valleys times their degeneracy, i.e., $\nu=2 \times 1+1 \times 3=5$. Keeping the same reasoning, one can predict using the fan chart the exact filling factors of all the quantum Hall regions at lower fields and, as a consequence, recover the unconventional filling factor sequence observed experimentally. As an example, the sudden transition between $\nu=6$ and $\nu=10$ at $B=11.1 \mathrm{~T}$ emanates from the simultaneous coincidence of one longitudinal and one oblique level at the Fermi energy.

By introducing a broadening parameter for the delocalized states $\Gamma_{\mathrm{dl}}=1 \mathrm{meV}$ and using the expressions of the conductivity tensor components, ${ }^{23,24}$ we have calculated the longitudinal and Hall resistances as a function of the magnetic field, and these are plotted in Fig. 1(c) (dotted and dashed lines, respectively). The comparison between the computed Hall resistance and the experimental one (thin solid line) shows a remarkably good agreement given the simplicity of the model used.

Finally, we note that due to the many-valley occupancy, an even filling factor does not necessarily correspond to an unpolarized state with the Fermi energy lying in a cyclotron gap. At $B=15 \mathrm{~T}$, for instance, one sees from Fig. 1(b) that four Landau levels are occupied, one spin-up and three spindown states (one of which is threefold degenerate), which implies that we have a partially polarized state at filling factor $\nu=6$.

\section{ROTATION}

Complementary measurements under tilted magnetic field have been carried out. For this purpose, the sample was mounted on a rotating stage, which enables us to tilt the sample in situ with respect to the applied external magnetic field, as represented schematically in the inset of Fig. 3(a). The longitudinal and inverse Hall resistances are plotted as a function of the perpendicular component of the magnetic field in Figs. 3(a) and 3(b) for different tilt angles $\theta$. The angles are determined precisely from the slope of the lowfield Hall resistance. We first note that at $\theta=0^{\circ}$ the magnetoresistance differs from the one shown in Fig. 1(a). This

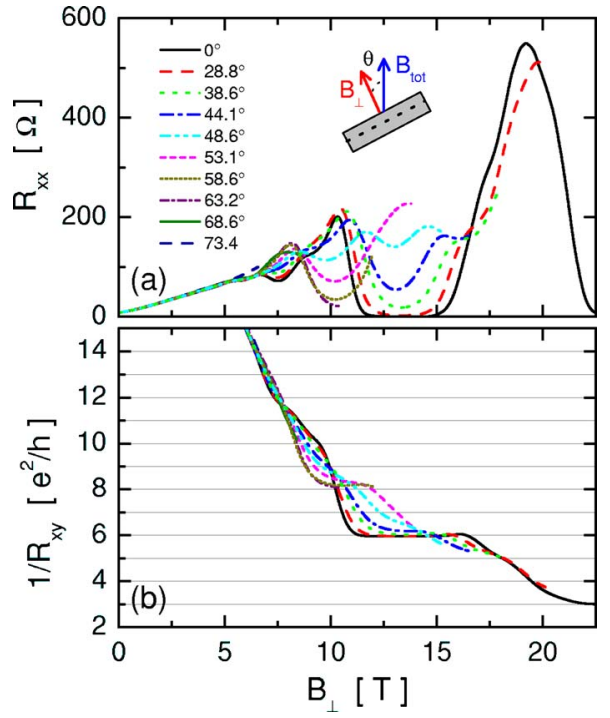

FIG. 3. (Color online) Longitudinal (a) and inverse Hall (b) resistances as a function of the perpendicular field for various tilt angles. A schematic of the setup of the rotation experiment is drawn in the inset, where $\theta$ is the angle between the applied field and the normal to the $2 \mathrm{D}$ plane.

results from the dependence of the carrier concentration, band structure, and band parameters on the cooling conditions, probably due to the thermal mismatch between the quantum-well structure and the $\mathrm{BaF}_{2}$ substrate. However, we are far from the strong deterioration of the electronic properties after several thermal cycles as reported in Olver et al. ${ }^{13}$ The large "intrinsic" carrier density, due to the Bi doping in our sample, will also tend to mask small "extrinsic" changes in carrier density after thermal cycling.

At first sight, the behavior of $R_{x x}$ versus $\theta$ is in agreement with the expected evolution of the density of states as a function of the tilt angle. We recall that, at fixed perpendicular magnetic field, the cyclotron energy remains constant while the Zeeman energy increases as the sample rotated away from the normal. In the simple case of one occupied subband, this effect leads to the closing (opening) of the energy gaps at even (odd) filling factors as a function of small increasing tilt angles. In our case, the complexity of the density of states, and above all the large number of parameters involved, makes the exact evolution of the levels versus $\theta$ impossible to predict with any confidence. Despite the large Landau-level broadening, it is nevertheless possible to see the closing and opening of gaps as the tilt angle increases. For example, at $B=13.3 \mathrm{~T}$ the longitudinal resistance minimum shrinks (the gap closes) while at $B=10.2 \mathrm{~T}$ or $B$ $=16.1 \mathrm{~T}, R_{x x}$ minima develop (a gap opens). Furthermore, from Fig. 3(b), one sees that the presence of new gaps for the latter field values corresponds to new integer filling factors, $\nu=8$ and $\nu=5$, respectively. This confirms that the consideration of only singly degenerate levels would be insufficient to explain these filling factor changes greater than 1. Thus, the tilted field resistance data clearly show the presence of levels of both longitudinal and oblique valleys in the density of states and the transfer of electrons between valleys with increasing tilt angle. 
A quantitative analysis is also precluded by the complex evolution of the fitting parameters involved with tilt angle. While the confinement and cyclotron effective masses remain constant, since the perpendicular component of the field, by definition, remains along the [111] direction, this is not the case for the electronic $g$ factors, since the projection of the ellipsoids along the $z$ axis (direction of the external field) varies with the tilt angle. For the initial configuration $z \|[111]$, the three oblique valleys are equivalent. However, this is no longer the case as soon as $\theta \neq 0^{\circ}$. The analysis is further complicated since to compute the tilt-angledependent $g$ factors, one should first know the orientation of each oblique valley with respect to the rotation plane. A simplified picture for the density of states of the oblique valleys at a fixed angle is the superimposition of three sets of spinsplit levels centered on the same Landau-level energies as they share a unique cyclotron mass. For small deviations between the $g$ factors, the three spin-split levels are energetically close to each other and may appear as a broadened level. Finally, we stress that a correct model for representing the density of states versus $\theta$ should include the well-known anticrossing of Landau levels originating from different subbands in the presence of an in-plane magnetic field. ${ }^{25}$

\section{CONCLUSION}

We have presented well resolved integer quantum Hall data for a narrow PbTe quantum well. Parallel conduction, which has plagued previous investigations, has been avoided by using $\mathrm{Pb}_{0.9} \mathrm{Eu}_{0.1} \mathrm{Te}$ barriers with a large Eu composition $(x=0.1)$. We are able to explain the unusual sequence of filling factors found in the experiments with a simple model, considering that three electrical subbands, two derived from the longitudinal valley and one derived from the oblique valleys, are occupied in the well. The PbTe system is rather unusual in that the large dielectric constant very efficiently screens the Coulomb potential of defects or impurities. This makes remote doping unnecessary in order to obtain high mobility samples. Future work will focus on trying to measure the integer, and possibly the fractional quantum Hall effect, using a quantum well with reduced carrier density with only one occupied longitudinal subband, for which a conventional sequence of filling factors is expected.

\section{ACKNOWLEDGMENT}

This work has been partially supported by a CNRS/CNPq exchange agreement and by FAPESP (Project 99/10359-7).
${ }^{1}$ H. Burkhard, G. Bauer, and W. Zawadzki, Phys. Rev. B 19, 5149 (1979).

${ }^{2}$ A. Krost, B. Harbecke, R. Faymonville, H. Schlegel, E. J. Fantner, K. E. Ambrosch, and G. Bauer, J. Phys. C 18, 2119 (1985).

${ }^{3}$ J. Singleton, E. Kress-Rogers, A. V. Lewis, R. J. Nicholas, E. J. Fantner, G. Bauer, and A. Otero, J. Phys. C 19, 77 (1986).

${ }^{4}$ S. Yuan, H. Krenn, G. Springholz, and G. Bauer, Phys. Rev. B 47, 7213 (1993).

${ }^{5}$ J. Oswald, P. Pichler, B. B. Goldberg, and G. Bauer, Phys. Rev. B 49, 17029 (1994).

${ }^{6}$ J. Oswald, B. B. Goldberg, G. Bauer, and P. J. Stiles, Phys. Rev. B 40, 3032 (1989).

${ }^{7}$ B. B. Goldberg, J. E. Furneaux, J. Oswald, G. Bauer, and P. J. Stiles, Semicond. Sci. Technol. 5, S151 (1990).

${ }^{8}$ G. Springholz, G. Bauer, and G. Ihninger, J. Cryst. Growth 127, 302 (1993).

${ }^{9}$ R. Dalven, in Solid States Physics, edited by H. Ehrenreich, F. Seitz, and D. Turnbull (Academic, New York, 1973), Vol. 28, p. 179.

${ }^{10}$ S. Yuan, H. Krenn, G. Springholz, Y. Ueta, G. Bauer, and P. J. McCann, Phys. Rev. B 55, 4607 (1997).

${ }^{11}$ A. Prinz, G. Brunthaler, Y. Ueta, G. Springholz, G. Bauer, G. Grabecki, and T. Dietl, Phys. Rev. B 59, 12983 (1999).

${ }^{12}$ G. Grabecki, J. Wrobel, T. Dietl, K. Byczuk, E. Papis, E. Kaminska, A. Piotrowska, G. Springholz, M. Pinczolits, and G. Bauer, Phys. Rev. B 60, R5133 (1999).

${ }^{13}$ M. M. Olver, J. Z. Pastalan, S. E. Romaine, B. B. Goldberg, G. Springholz, G. Ihninger, and G. Bauer, Solid State Commun.
89, 693 (1994).

${ }^{14}$ G. Springholz, G. Ihninger, G. Bauer, M. M. Olver, J. Z. Pastalan, S. Romaine, and B. B. Goldberg, Appl. Phys. Lett. 63, 2908 (1993).

${ }^{15}$ A. M. P. dos Anjos, E. Abramof, P. H. O. Rappl, and H. Closs, Braz. J. Phys. 34, 653 (2004).

${ }^{16}$ G. Bauer, H. Pascher, and W. Zawadzki, Semicond. Sci. Technol. 7, 703 (1992).

${ }^{17}$ E. Abramof, E. A. de Andrada e Silva, S. O. Ferreira, P. Motisuke, P. H. O. Rappl, and A. Y. Ueta, Phys. Rev. B 63, 085304 (2001).

${ }^{18}$ I. I. Zasavitskii, E. A. de Andrada e Silva, E. Abramof, and P. J. McCann, Phys. Rev. B 70, 115302 (2004).

${ }^{19}$ D. L. Mitchell and R. F. Wallis, Phys. Rev. 151, 581 (1966).

${ }^{20}$ H. Pascher and G. Bauer, in High Magnetic Fields in Semiconductor Physics, edited by G. Landwehr (Springer, Berlin, 1987), p. 179.

${ }^{21}$ Erasmo A. de Andrada e Silva, Phys. Rev. B 60, 8859 (1999).

${ }^{22}$ S. Yuan, G. Springholz, G. Bauer, and M. Kriechbaum, Phys. Rev. B 49, 5476 (1994).

${ }^{23}$ T. Ando, A. B. Fowler, and F. Stern, Rev. Mod. Phys. 54, 437 (1982).

${ }^{24}$ M. van der Burgt, V. C. Karavolas, F. M. Peeters, J. Singleton, R. J. Nicholas, F. Herlach, J. J. Harris, M. V. Hove, and G. Borghs, Phys. Rev. B 52, 12218 (1995).

${ }^{25}$ G. Bastard, Wave Mechanics Applied to Semiconductor Heterostructures (Editions de Physique, Paris, 1988). 